\title{
Impact of Type II Spicules in the Corona: Simulations and Synthetic Observables
}

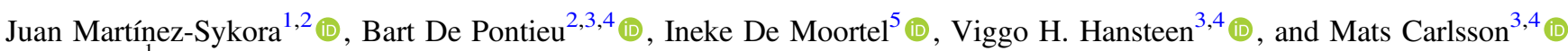 \\ ${ }^{1}$ Bay Area Environmental Research Institute, NASA Research Park, Moffett Field, CA, CA 94952, USA; juanms@1msal.com \\ ${ }^{2}$ Lockheed Martin Solar and Astrophysics Laboratory, Palo Alto, CA 94304, USA \\ ${ }^{3}$ Institute of Theoretical Astrophysics, University of Oslo, P.O. Box 1029 Blindern, NO-0315 Oslo, Norway \\ ${ }^{4}$ Rosseland Centre for Solar Physics, University of Oslo, P.O. Box 1029 Blindern, NO-0315 Oslo, Norway \\ ${ }^{5}$ School of Mathematics and Statistics, University of St. Andrews, St. Andrews, Fife, KY16 9SS, UK \\ Received 2017 December 19; revised 2018 April 11; accepted 2018 May 2; published 2018 June 19
}

\begin{abstract}
The role of type II spicules in the corona has been a much debated topic in recent years. This paper aims to shed light on the impact of type II spicules in the corona using novel 2.5D radiative MHD simulations, including ionneutral interaction effects with the Bifrost code. We find that the formation of simulated type II spicules, driven by the release of magnetic tension, impacts the corona in various manners. Associated with the formation of spicules, the corona exhibits (1) magneto-acoustic shocks and flows, which supply mass to coronal loops, and (2) transversal magnetic waves and electric currents that propagate at Alfvén speeds. The transversal waves and electric currents, generated by the spicule's driver and lasting for many minutes, are dissipated and heat the associated loop. These complex interactions in the corona can be connected with blueshifted secondary components in coronal spectral lines (red-blue asymmetries) observed with Hinode/EIS and SOHO/SUMER, as well as the EUV counterpart of type II spicules and propagating coronal disturbances observed with the $171 \AA$ and $193 \AA$ SDO/AIA channels.
\end{abstract}

Key words: magnetohydrodynamics (MHD) - methods: numerical - radiative transfer - Sun: atmosphere - Sun: corona

Supporting material: animations

\section{Introduction}

Highly dynamic chromospheric jets that permeate the corona are one of the most common features observed at the solar limb. Consequently, given their occurrence rate, they have been suggested as contributors to coronal heating and/or as possible drivers of the solar wind (e.g., Beckers 1968; Athay \& Holzer 1982; De Pontieu et al. 2011; McIntosh et al. 2011; De Pontieu et al. 2017). When seen at the limb, such jets are named spicules. Two types have been observationally identified (De Pontieu et al. 2007a; Tsiropoula et al. 2012; Pereira et al. 2014): type I spicules are observed to have relatively slow rise velocities and small heights, type II spicules appear to rise much more rapidly and often extend to heights of at least $>10 \mathrm{Mm}$. However, since the exact nature of type II spicules has been unknown (Martinez-Sykora et al. 2017), their mass and energy contribution to the corona has so far not been properly addressed. Hence, whether (and how much) type II spicules contribute or impact the corona and/or solar wind is under ongoing, vigorous debate (e.g., compare with Madjarska et al. 2011; Klimchuk 2012).

Spicules are an observational phenomenon in which many different complex physical processes appear to be at work. In the current paper, we take a broad approach to the problem of understanding coronal heating associated with spicules. We do not limit ourselves to a simplifying scenario in which ad-hoc advection of expanding gas along a single field line dominates the energetics of spicules (Klimchuk 2012; Klimchuk \& Bradshaw 2014). Instead, our research takes a broader approach by studying the coronal heating associated with self-consistently generated features that show many similarities with observed properties of spicules by using a $2.5 \mathrm{D}$ radiative MHD numerical model. This model includes ambipolar diffusion, which is a key process for the formation of type II spicules (Martinez-Sykora et al. 2017). We thus continue the solar physics community's quest to understand how much coronal heating is associated with spicules (see also, e.g., Tsiropoula et al. 2012; Pereira et al. 2014, and references therein). Some known observational constraints and properties of type II spicules are listed as follows. These jets reach velocities of [40-150] $\mathrm{km} \mathrm{s}^{-1}$ and heights of $\sim 10 \mathrm{Mm}$ prior to falling back some 3-10 minutes after their first appearance (Pereira et al. 2014; Skogsrud et al. 2015). In contrast to type I spicules, some of the ejected chromospheric plasma of type II spicules is often heated to transition region (TR) temperatures. Therefore, these spicules observed in the chromospheric Ca II $\mathrm{H}$ line, e.g., with the Solar Optical Telescope on board Hinode (SOT, Tsuneta et al. 2008), are typically short lived (1-2 minutes), whereas type II spicules observed in hotter or more opaque chromospheric lines such as Mg II or TR lines such as Si IV observed with IRIS (De Pontieu et al. 2014b), are longer lived (3-10 minutes).

Type II spicules have been associated with various TR and coronal observables. Their on-disk counterparts, so-called Rapid Blue-shifted Events (RBEs), are associated with brightenings in EUV spectral lines that are sensitive to plasma at TR and coronal temperatures (De Pontieu et al. 2011) as observed with the Atmospheric Imaging Assembly (AIA, Lemen et al. 2012) on board the Solar Dynamic Observatory (SDO) (but consider also Madjarska et al. 2011). Transition region and coronal spectral lines observed by the Extreme-ultraviolet Imaging Spectrometer (EIS, Culhane et al. 2007) on board Hinode show blueshifted secondary components that could be associated with type II spicule dynamics (e.g., De Pontieu et al. 2009; McIntosh \& De Pontieu 2009a; Bryans et al. 2010; Peter 2010). These features have nevertheless not been conclusively connected to type II spicules. More recently, Propagating Coronal Disturbances (PCDs) have been linked to type II spicules (De Pontieu et al. 2017). Their observations 
show that coronal loops are formed and exist for many tens of minutes in association with spicules. To interpret these observations, recent models of type II spicules (MartinezSykora et al. 2017) were used. This radiative MHD model, based on the Bifrost code (Gudiksen et al. 2011), selfconsistently generates jets that resemble type II spicules. In the current paper, we study in greater detail the impact of these simulated type II spicules on the corona. Our results show that the complexity of our 2.5D MHD model invalidates many of the simplifying assumptions made in previous modeling attempts to address the coronal impact of type II spicules (e.g., compare the results of this paper with those of Klimchuk \& Bradshaw 2014 and Petralia et al. 2014).

Type II spicules are also associated with various types of waves that could, in principle, also contribute to the driving of the solar wind and heating of the corona. Spicules show torsional (e.g., De Pontieu et al. 2012, 2014a) and both low and high frequency transversal motions (De Pontieu et al. 2007b; Okamoto \& De Pontieu 2011; Srivastava et al. 2017). These studies provide estimates of the wave energy associated with spicules and it is claimed that waves carry enough energy flux into the corona to power the solar wind (De Pontieu et al. 2007b; McIntosh et al. 2011). However, it is unclear how this wave energy is generated and/or dissipated. Until now, we have not had access to realistic models for the generation of magnetic waves in spicules. In this paper, we use the MartinezSykora et al. (2017) model to shed some light on these issues.

Our paper starts with a description of the numerical models (Section 2). Section 3 details our results where we first describe TR and coronal synthetic observables associated with the type II spicules (Section 3.1), and determine the width of the coronal loops formed in association with the spicules (Section 3.1.1). We also analyze the flows and shocks that penetrate into the corona (Section 3.2) as well as the mass and energy deposited into the corona (Section 3.3). We investigate the causes for the widths of the spicule-associated coronal loops and also the heating distribution along the loops (Section 3.3.1 and Section 3.4). We finish with a discussion and conclusions (Section 4).

\section{Simulation}

We use two types of $2.5 \mathrm{D}$ radiative $\mathrm{MHD}$ numerical simulations, both based on the Bifrost code (see Gudiksen et al. 2011 for details on the numerical implementation and validation). The numerical methods implemented in our simulations include radiative transfer with scattering (Skartlien 2000; Hayek et al. 2010; Carlsson \& Leenaarts 2012), and thermal conduction along the magnetic field. The numerical domain in both simulations is identical and covers a region from the upper layers of the convection zone (down to $3 \mathrm{Mm}$ below the photosphere) into the corona (up to $40 \mathrm{Mm}$ above the photosphere). The spatial resolution along the vertical axis is nonuniform and ranges from $12 \mathrm{~km}$ (from the convection zone to the TR) slowly increasing with height to $69 \mathrm{~km}$ (in the corona). The domain extends $96 \mathrm{Mm}$ in the horizontal direction with a spatial resolution of $14 \mathrm{~km}$. The energy flux entering the bottom of the computational domain and the chemical composition of the model is set to solar values. The only free parameter in these simulations is the initial distribution of the magnetic field. In both cases, there is no new magnetic flux injected at the lower boundary and we have seeded two main opposite polarities of plage or enhanced network with an unsigned magnetic field of $190 \mathrm{G}$. These regions are connected by $\sim 50 \mathrm{Mm}$ long loops. In particular, in both of these models, the hot corona is self-consistently maintained, despite the constraints inherent in $2.5 \mathrm{D}$ dynamics. Previous 2D simulations with a smaller numerical domain required the addition of a "hot plate" at the top boundary to maintain coronal temperatures due to their smaller numerical domain and/or lack of ability to resolve various small-scale processes (Martínez-Sykora et al. 2017).

In the "Generalized Ohm's Law" (GOL) simulation, we have included the effects of interactions between ions and neutrals in the partially ionized chromosphere by including ambipolar diffusion through using a GOL in the induction equation (Cowling 1957; Braginskii 1965). In this simulation, jets that resemble type II spicules are formed ubiquitously, as a result of ambipolar diffusion (e.g., see the example shown with the arrow in the left panel in Figure 1). In contrast, simulations without ambipolar diffusion, i.e., the "non-GOL" simulation in Martínez-Sykora et al. (2017), as well as previous models (Martínez-Sykora et al. 2013; Carlsson et al. 2016), typically do not produce type II spicules, or if at all, only rarely. Unless otherwise noted, we will refer to the GOL simulation when describing the impact of type II spicules on the corona. Figure 1 shows that the chromospheric jet and associated hot loop are not always perfectly aligned along the same field lines. This is because the various contributing mechanisms for the heating and flows (i.e., currents, waves, flows, and fluxes) do not always occur along exactly the same magnetic field line. Instead, they occur on a range of magnetic field lines that either pass through or occur in a close vicinity of the chromospheric spicule. These field lines are all coupled together and connected to the driving mechanism that generates chromospheric spicules. The varying spatial relationship between the currents and flows is caused by variations in which field lines release tension and carry currents (see also Martinez-Sykora et al. 2017). The spatial offsets between the various contributing mechanisms are quite small and difficult to resolve at the spatial resolution of current coronal instruments ( $>1$ arcsec).

We briefly summarize the main relevant results on the generation of type II spicules (see Martinez-Sykora et al. 2017 for details): simulated type II spicules are driven by at least two different processes. (1) One process is the release of magnetic energy, which has been built up by convective motions near magnetic flux concentrations, such as in network or plage regions. In such regions, the convective motions can impose substantial tension on the magnetic field. This tension often "diffuses" away from regions where the gas pressure dominates (i.e., where plasma beta, $\beta>1$ ), toward regions where $\beta \approx 1$, higher in the atmosphere. Ambipolar diffusion is the process that helps to move this magnetic field through the photosphere and lower chromosphere. At the same time, ambipolar diffusion helps to spatially confine the magnetic tension to thin layers. When this magnetic field eventually reaches heights where $\beta \approx 1$, the magnetic tension is released in a similar manner as the whiplash effect, which gives rise to pressure gradients and thereby accelerates plasma. (2) The other process is when magneto convective motions perturb the overlying and highly bent magnetic field, producing magnetoacoustic waves traveling almost parallel to the surface. Once the shock reaches the highly bent magnetic field at heights where $\beta \approx 1$, the wave mode changes, and in combination with the release of some of the tension from the highly bent field lines, leads to the 

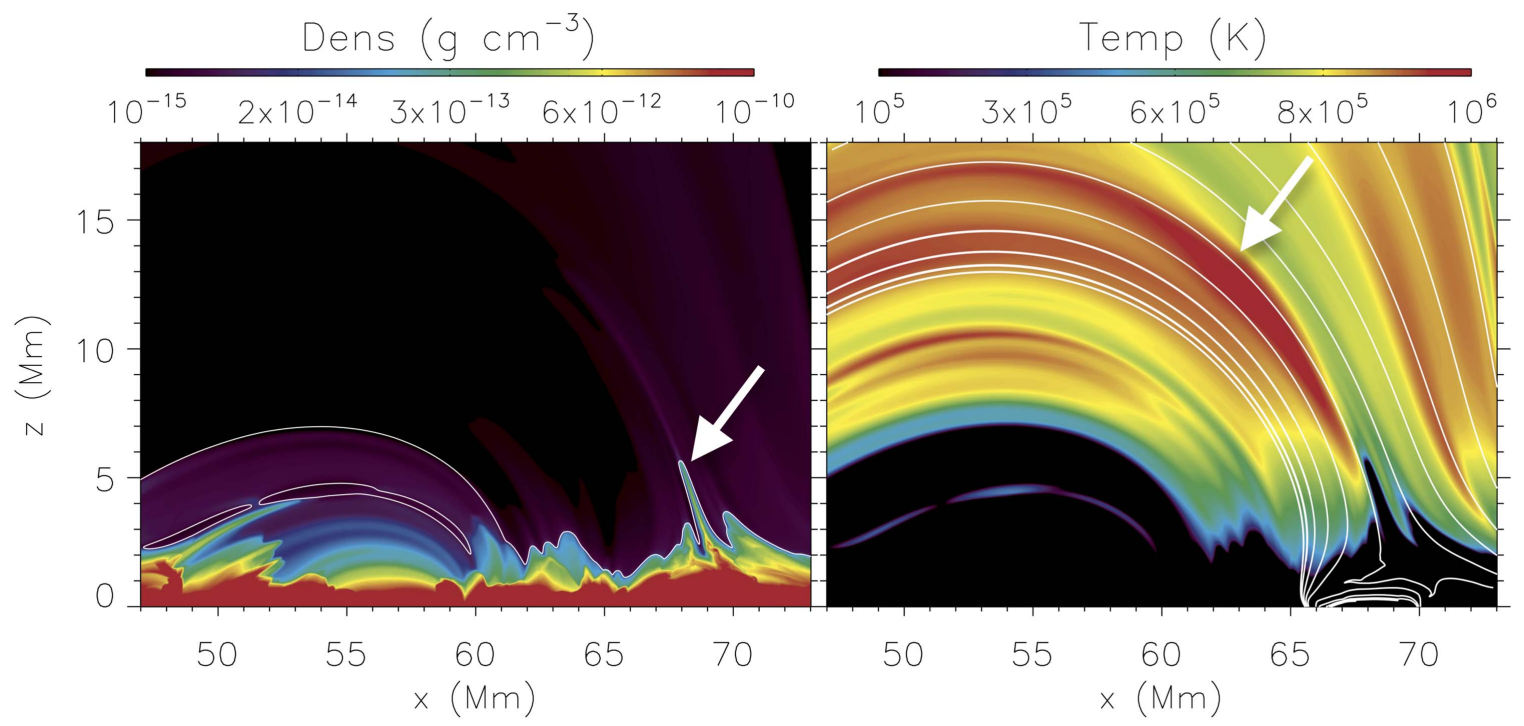

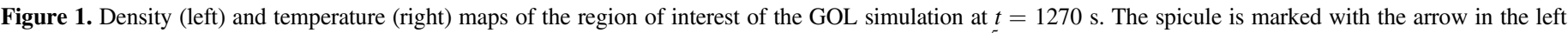

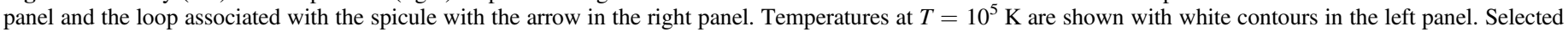
field lines are shown in the right panel in white.

formation of the jet and Alfvénic waves. The example shown in this work (Figure 1) is this second case. In both cases, the formation mechanism causes a magnetoacoustic shock, which in turn produces chromospheric jets, reaching velocities of $50-100 \mathrm{~km} \mathrm{~s}^{-1}$. These shocks pass through the chromosphere in only a few tens of seconds. The chromospheric signature of these shocks is found as short-lived RBEs in the line wings of, e.g., $\mathrm{H} \alpha$ and $\mathrm{Ca}$ II (Rouppe van der Voort et al. 2009). In addition to magnetoacoustic shocks, the release of magnetic tension and mode conversion drive high-frequency transversal waves that propagate into the corona. Within a timespan of a few minutes, cool chromospheric ejected material is heated by the dissipation of currents via ambipolar diffusion to TR temperatures, in broad agreement with the observations of Pereira et al. (2014) and Skogsrud et al. (2015). The coronal impact of the spicules is the same for both processes that drive the spicules.

\section{Results}

An obvious outstanding question to the study of MartinezSykora et al. (2017) addressed in this paper is, what is the impact of type II spicules on the corona? Before describing our results, we list the TR and coronal synthetic observables of the simulated type II spicules to see whether they fulfill the constraints derived from observations, such as those listed in the Introduction (see also De Pontieu et al. 2017). For this, we calculate synthetic TR (C IV $1548 \AA$ ) and coronal spectral profiles (Fe X $184 \AA$ A, Fe XII $195 \AA$, and Fe XIV $211 \AA$ ), assuming ionization in statistical equilibrium, the optically thin approximation as in Hansteen et al. (2010) and coronal abundances (Schmelz et al. 2012). These profiles have been integrated along the vertical axis, i.e., as seen from above. Additionally, we have degraded the synthetic profiles to the Hinode/EIS spatial ( $1^{\prime}$ per pixel) and spectral resolution ( $\sim 25 \mathrm{~km} \mathrm{~s}^{-1}$ ) (Figure 2).

\subsection{Observables and Constraints}

Many observables are associated with the type II spicules in all of the synthesized TR and coronal spectral lines. This is illustrated with Figure 2, which shows the total line intensity, the Doppler shift, the line width, and the RB-asymmetry in the four rows from top to bottom.

At the beginning of the chromospheric ejection, all four synthesized spectral lines show a brightening in intensity (top row, $x=68 \mathrm{Mm}, t=1210 \mathrm{~s}$ ). In the particular case shown here, the synthetic Fe XIV $211 \AA$ intensity is too faint to be observed with Hinode/EIS, i.e., only $\sim 5 \times 10^{-3} \mathrm{DN} \mathrm{s}^{-1}$ (panel (D)). On the other hand, C IV has enough intensity to be observed with $\mathrm{SOHO} / \mathrm{SUMER}$ and Fe X $184 \AA$ and Fe XII $195 \AA$ with Hinode/EIS as long as the observations are integrated over exposure times of $\sim 1,5$, and $>30$ seconds or more, respectively (panels $(\mathrm{A})-(\mathrm{C})$ ). The various simulated spicules provide a range of intensities at the different wavelengths, and in some cases we find stronger Fe XII intensities (up to $300 \mathrm{erg} \mathrm{cm}^{-2} \mathrm{~s}^{-1} \mathrm{sr}^{-1}$ ) and weaker Fe X than the example shown here. These values are comparable to those in QS by Brooks et al. (2009) and Mariska (2013)

Looking at the intensity observables in the coronal lines (Fe XII and Fe XIV, panels (C) and (D) in Figure 2), one can distinguish two different brightenings, or PCDs, propagating in time and associated with the spicules. One of them is a "slow" PCD, which at $t=1210 \mathrm{~s}$ is at $x=68 \mathrm{Mm}$ and at $t=1300 \mathrm{~s}$ is at $x=60 \mathrm{Mm}$ (see the arrow in panel (C)). The other one is faster, and appears as an almost horizontal intensity brightening in Fe XII and Fe XIV at $t=1230 \mathrm{~s}$ in the region $x=[58$, $68 \mathrm{Mm}$ (arrow in panel (D)). As described in detail in Sections 3.2 and 3.3, these observables are a consequence of a combination of a magneto-acoustic shock, (real) flows, and heating processes. Further details of the synthetic PCD observables are discussed and used to interpret SDO/AIA observations in De Pontieu et al. (2017).

The synthetic coronal and TR Doppler shifts show only minimal changes (second row) in the spicule location, with the exception of C IV and Fe XIV, which show blueshifted profiles at the start of the chromospheric ejection. The lack of Doppler shift variation for Fe X or very weak Doppler shift variation for Fe XII are due to the line-of-sight integration and also partially due to the relatively low spatial resolution of the Hinode/EIS 

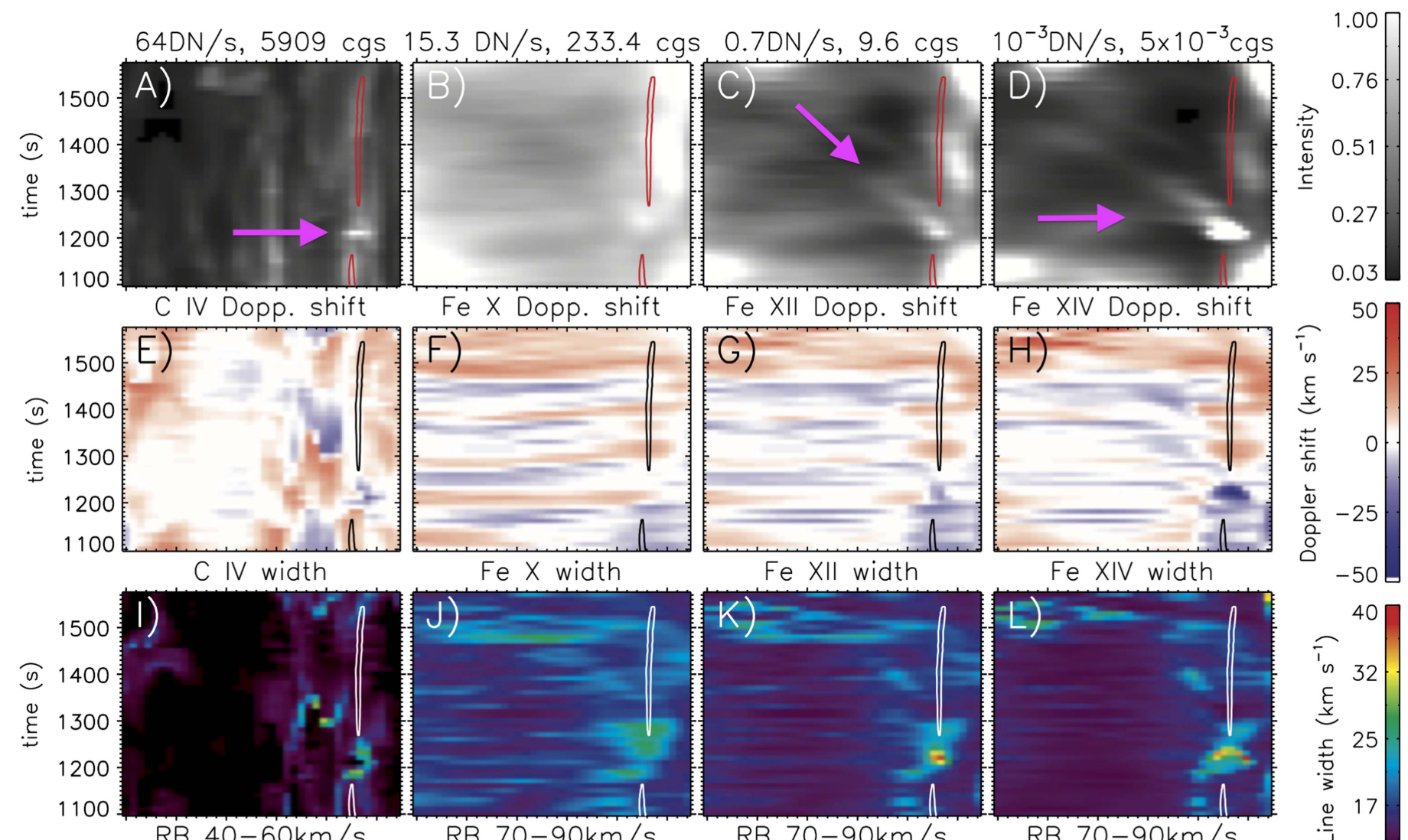

Fe XII width
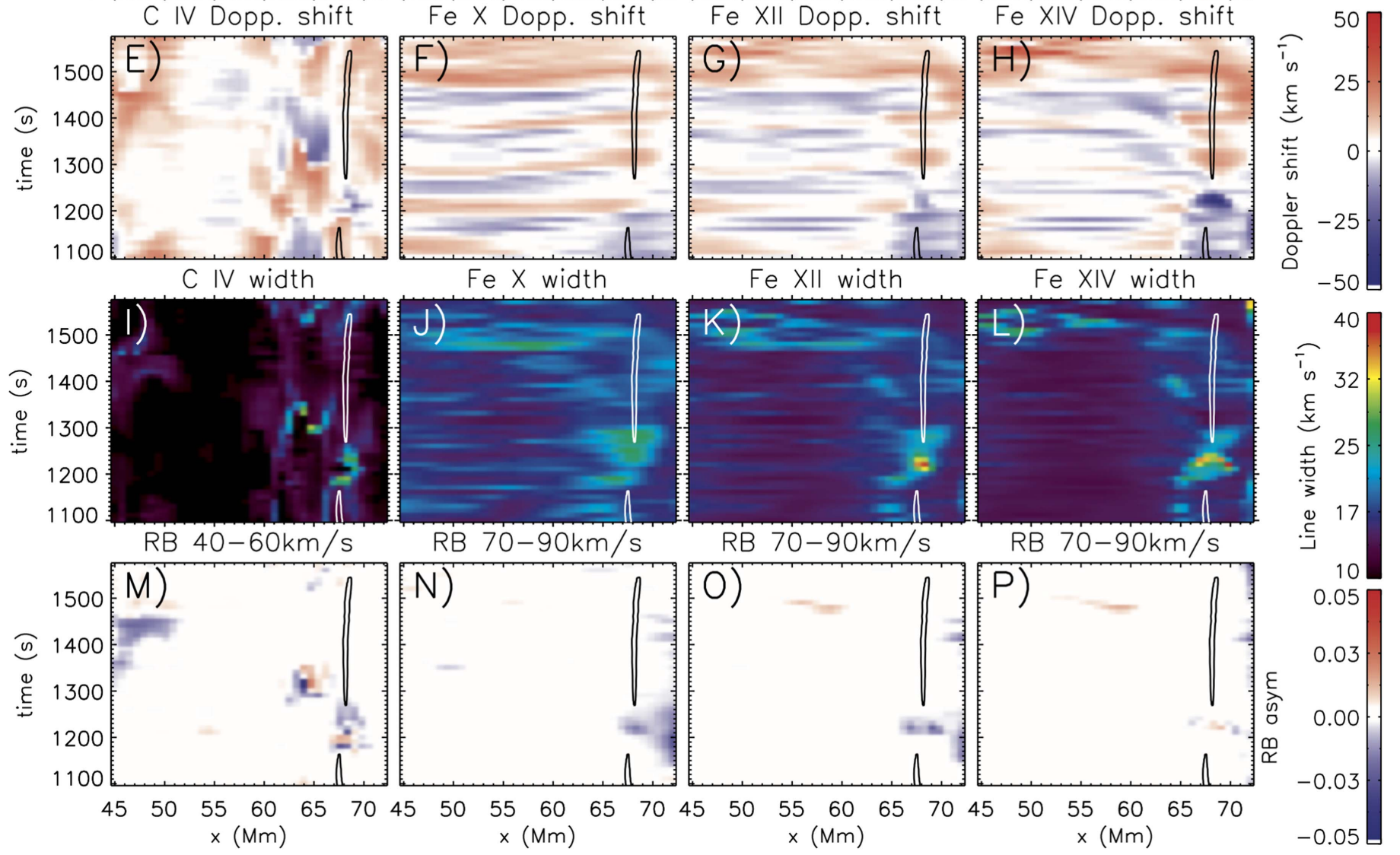

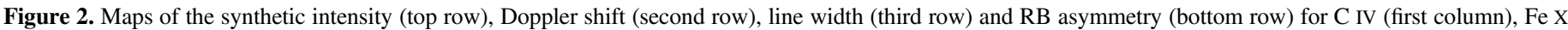

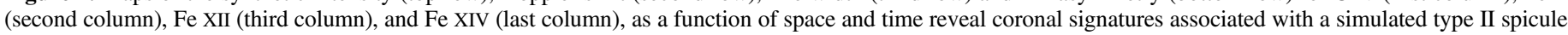

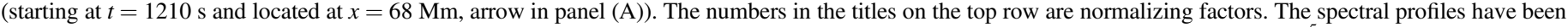

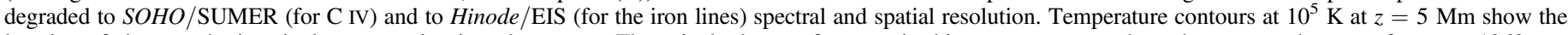

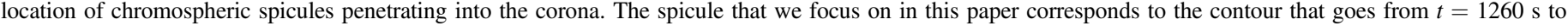
$t=1550 \mathrm{~s}$ (Figure 1).

instrument. Consequently, there is more background emission in the corona along the line of sight so that the Doppler shifts are dominated by the background. In this particular simulated spicule most of the Fe XIV $211 \AA$ synthetic intensity (Figure 2) comes from the spicule and very little, if any, is from the overlying and surrounding plasma not associated with the spicules due to the fairly low coronal temperature in this model. Consequently, the synthetic Doppler shift is derived for the most part from the spicular velocities and does not have other possible contributions. Similarly to the synthetic Si IV shown in Martinez-Sykora et al. (2017) the spicule provides a very large contribution to the synthetic intensity, despite the LOS integration and pixel resolution. Therefore, we find a large signal in the C IV spectral lines (and in general for all TR spectral lines), in both Doppler shift and red-blue (RB) asymmetries. In the bottom row of Figure 2, we present the RB asymmetries calculated following the same approach as that used by De Pontieu et al. (2009) which interpolates in the wavelength axis using spline. We tested the Klimchuk et al. (2016) interpolation method (not shown here), and the calculated RB asymmetries do not differ from the one using spline interpolation shown in Figure 2.

All the spectral profiles become up to two times broader compared to their surroundings at the location of spicule acceleration (third row). Similarly, observations also show nonthermal broadening in spicules of EUV spectral lines (e.g., McIntosh \& De Pontieu 2009a).

Despite the low resolution of Hinode/EIS, one can still capture small-scale flows by applying RB asymmetry analysis (e.g., De Pontieu et al. 2009; McIntosh \& De Pontieu 2009a) or double Gaussian fitting (Peter 2010). As for Fe XIV, RB asymmetries in Figure 2, for this particular case, are very small since most of the emission is very localized within the associated spicular plasma. The synthetic Fe X and Fe XII 


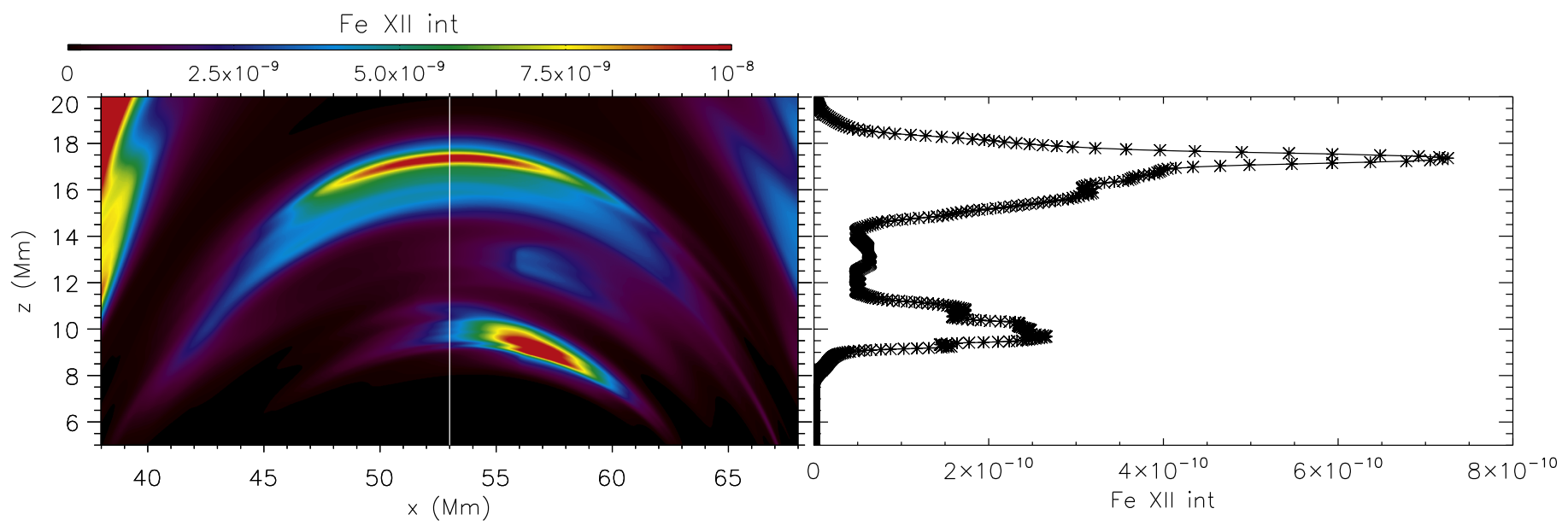

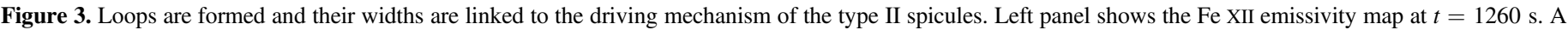
vertical cut of Fe XII emissivity at the apex of the loops (white vertical line in the left panel) is shown in the right panel. Asterisks indicate grid cell positions.

spectral profiles reveal a blueshifted secondary component at the location of the type II spicule and, for the first time, the synthetic RB asymmetries $\sim 5 \%$ are roughly in agreement with observations (e.g., De Pontieu et al. 2009; Peter 2010). Indeed, previous models did not reproduce sufficient RB asymmetries ( $\sim .1 \%$ Martínez-Sykora et al. 2011) due to the lack of type II spicules. These RB asymmetries are a clear signal of mass and energy injected into the corona due the combination of a magnetoacoustic shock and real flows, as detailed in Section 3.2.

The second spectral component in the TR line (C IV, panel (M) in Figure 2) is found at lower velocities [40-60 km s${ }^{-1}$ ] as compared to the coronal lines [70-90 $\mathrm{km} \mathrm{s}^{-1}$ ] (Fe X, and Fe XII, panels $(\mathrm{N})$ and $(\mathrm{O}))$. This is due to the acceleration of the plasma as it expands into the corona. This synthetic observational property and the measured speeds are in agreement with observations (McIntosh \& De Pontieu 2009b). We took into account spatial resolution and instrumental broadening of the spectral lines since they impact the intensity and location of the secondary component (Martínez-Sykora et al. 2011).

Could spicules provide enough coronal emission to give any signal in the coronal SDO/AIA channels? The synthetic Fe X $184 \AA$, Fe XII $193 \AA$, and Fe XIV $211 \AA$ intensities associated with the spicule are roughly 600,6 , and $10^{-1} S D O /$ AIA $\mathrm{DN} \mathrm{s}^{-1}$, respectively. Consequently, the simulated spicule provides enough synthetic intensity contribution from Fe X $184 \AA$ to be easily observed in the 171 channel. For the 193 SDO/AIA channel, the Fe XII $193 \AA$ intensity coming from the spicule is much smaller than from Fe X. According to these results, it is very unlikely to see any Fe XII contribution coming from the spicule in the 193 SDO/AIA channel where a typical coronal hole and quiet Sun produce something like 9 and $100 \mathrm{DN} \mathrm{s}^{-1}$, respectively. Despite this, running differences should be able to reveal the intensity variations coming from Fe XII $193 \AA$. This is in accordance with De Pontieu et al.'s (2011) conclusions. Finally, synthetic Fe XIV $211 \AA$ intensity coming from the spicule (and any in this simulation) is too small to provide an appreciable signal in SDO/AIA channel $211 \AA$ A. However, this model has a cool corona for an active region and is limited to a very specific field configuration, note that other more active regions reveal RB-asymmetries in hot lines such as Fe XIV (De Pontieu et al. 2009).

\subsubsection{Loop Width}

Recent observations with Hi-C (Kobayashi et al. 2014) suggest that we may resolve the width of many of the coronal loops ( 500-1500 km Peter et al. 2013; Aschwanden \& Peter 2017), which are comparable to photospheric granulation scales $(\sim 500 \mathrm{~km})$ (Abramenko et al. 2012). This, according to Aschwanden \& Peter (2017), suggests that the heating mechanism in the corona is driven by macro-scales instead of unresolved micro-physics. In addition, observed spicules also seem to have this typical width.

Figure 3 shows a map of the Fe XII emissivity (left) and a vertical cut through a loop (right). The right panel shows that the width of the loops ${ }^{6}$ associated with the spicules is well resolved, with more than 20 grid points across the loop. ${ }^{7}$ The typical width of loops associated with the simulated spicules ranges between 300 and $900 \mathrm{~km}$. The formation of type II spicules is associated with a PCD and the formation of coronal loops (De Pontieu et al. 2017). The loops associated with the simulated spicules of this paper have similar widths to the observations reported above (of the order of a few hundreds of kilometers). As described in the following sections, the width of the loops associated with the simulated spicules is determined primarily by the driving mechanism that generates flows in deeper layers, the magnetic connectivity, and heating within the magnetic field linked with the spicule (detailed in the following sections). The artificial magnetic diffusion or viscosity plays a minor role in the loop width: in our model the typical dissipation and viscous lengths are five grid points, whereas the loop contains between 20 and 100 grid points along the vertical axis (as seen in Figure 3), and even more in the horizontal axis.

\subsection{Flows and Shocks}

The dynamics occurring in the corona are a consequence of both magnetoacoustic shocks and flows: the simulated type II spicules drive magnetoacoustic shocks and flows, propagating along the magnetic field at speeds of the order of $100-200 \mathrm{~km} \mathrm{~s}^{-1}$, due to the release of magnetic tension, in a

\footnotetext{
6 In the following, we use the term "loop" to refer to a region of magnetic field lines that have a common energy and flow source.

7 Note that the horizontal axis has a smaller spatial resolution $(14 \mathrm{~km})$ than the vertical axis in the corona $(20-50 \mathrm{~km})$.
} 
manner similar to the whiplash effect, or due to a wave mode conversion driven by photospheric motions. In principle, if spicules are driven only by acoustic shocks, they must satisfy the Rankine-Hugoniot (RH) conditions which, in a frame of reference moving with the shock front, are given by

$$
\begin{aligned}
\frac{\rho_{\text {post }}}{\rho_{\text {pre }}} & =\frac{(\gamma+1) M_{\mathrm{pre}}^{2}}{2+(\gamma-1) M_{\mathrm{pre}}^{2}} \\
\frac{u_{\mathrm{post}}}{u_{\mathrm{pre}}} & =\frac{2+(\gamma-1) M_{\mathrm{pre}}^{2}}{(\gamma+1) M_{\mathrm{pre}}^{2}} \\
\frac{e_{\mathrm{post}}}{e_{\mathrm{pre}}} & =\frac{2 \gamma M_{\mathrm{pre}}^{2}-(\gamma-1)}{(\gamma+1)},
\end{aligned}
$$

where $\rho, u, e$, and $\gamma$ have their usual meanings. The subscripts pre and post refer to pre- and post-shock plasma, respectively (Priest 1982). $M$ is the Mach number $\left(M=u / c_{s}\right)$ and the sound speed is $c_{s}^{2}=\gamma P / \rho$. Note that these equations assume no entropy sources outside the shock discontinuity as well as including only the pressure gradient force, i.e., a hydrodynamic fluid without external forces, or the Lorentz force.

The example shown in Figures 1 and 2 is examined in detail in Figure 4, where panel (A) shows the density stratification and with a density jump located at $[x, z]=[65,11] \mathrm{Mm}$ (see the arrows). The jump is easier to discern in the parallel velocity map (along the magnetic field) shown in panel (B). Taking into account the coronal sound speed $\left(\sim 150 \mathrm{~km} \mathrm{~s}^{-1}\right.$, panel (C)) and the fact that the shock travels through the corona at $150 \mathrm{~km} \mathrm{~s}^{-1}$, the RH relations (right-hand side of Equations (1)-(2)) can be calculated from the velocity along the magnetic field (panel (B)) subtracting the front shock speed $\left(150 \mathrm{~km} \mathrm{~s}^{-1}\right)$, sound speed (panel (C)), and the adiabatic parameter $\gamma(1.66)$ shown in panels (D) and (E). From there, one would predict a density jump of 1.4 and a velocity ratio of 0.6 (panels (D) and (E)). However, the jumps in the corona are of the order of 1.8 and 0.75 for the density and velocity, respectively (panels (A) and (B)). This disagreement is due to the fact that these jumps depend not only on the gradient of pressure but also on the Lorentz force, which is absent in 1D hydro-models, and other entropy sources working on the bulk flow, in particular, some of the plasma moving with the shock wave is heated via Joule heating and/or thermal conduction and thus becomes part of the corona. Note that this perturbation (or jump) will travel $1 \mathrm{Mm}$ in $10 \mathrm{~s}$. This explains (1) the short-lived brightening in the AIA channels due the increase in density; (2) the nonthermal line broadening and RB asymmetries in the TR and coronal spectral lines at the beginning of the spicule formation are due to the velocity jump and the difference in velocity between the loop associated with the spicule and its surroundings; (3) finally, this perturbation, which travels along the loop, leads to the synthetic "slow" PCD.

\subsection{Mass and Energy Deposition in Association with Spicules}

The mass and energy deposition in the corona from a spicule results from a combination of shocks, flows, Joule heating, and thermal conduction. The plasma is heated along the loop through Joule heating (see below), resulting from numerical diffusivity. The artificial diffusion will not impact the amount of Joule heating dissipation (Galsgaard\& Nordlund 1996; Abbett et al. 2000; Peter et al. 2004; Gudiksen \& Nordlund 2005a, 2005b;
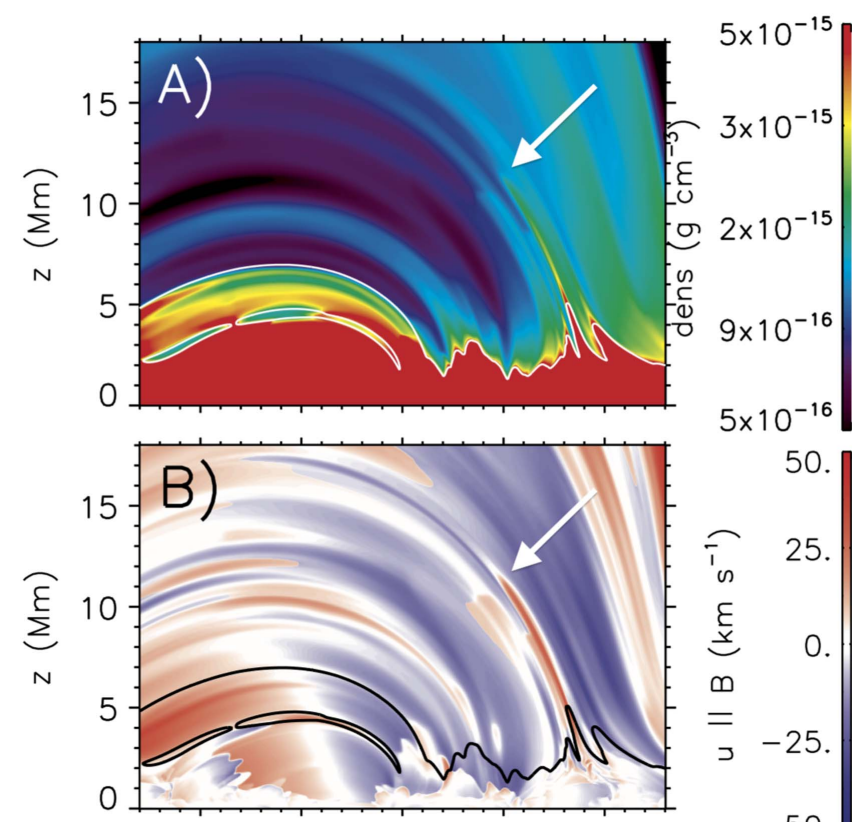

50

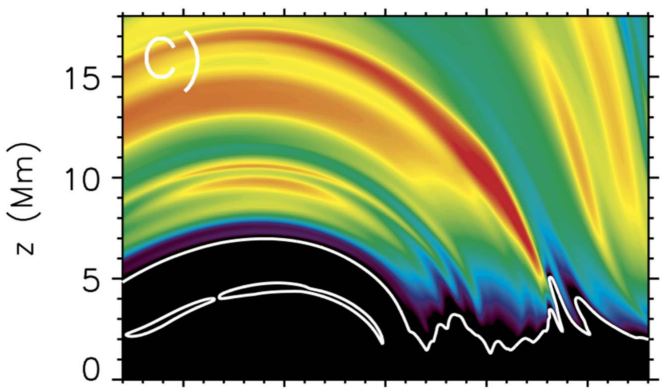

in

$\stackrel{\varepsilon}{\underline{\varepsilon}}$

m

$=$

$-25$

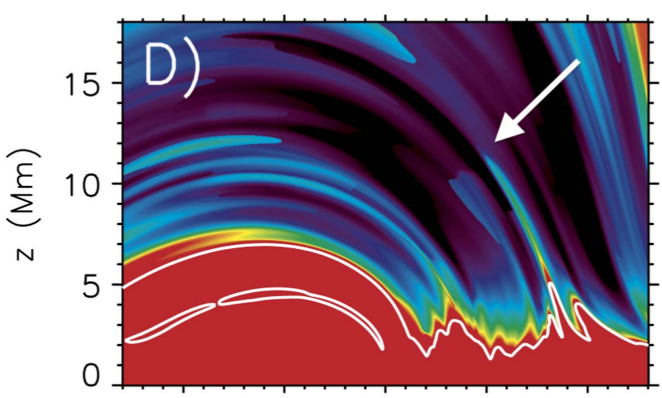

$-50$.

150.

$\widetilde{T}$

138.

E 125

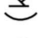

o

112.

100.

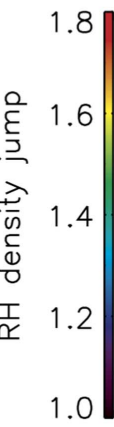

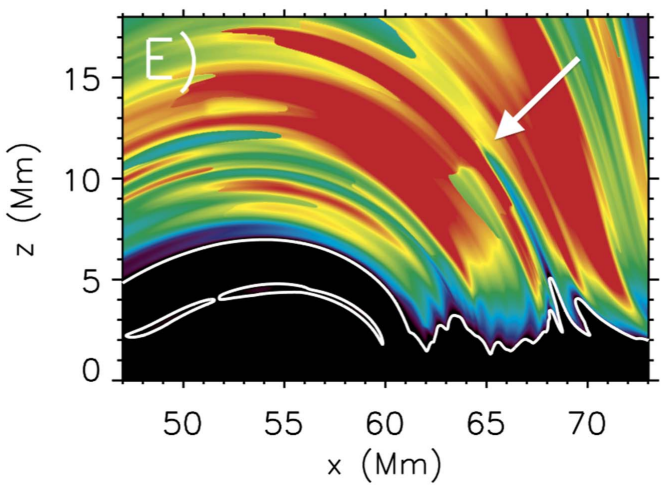

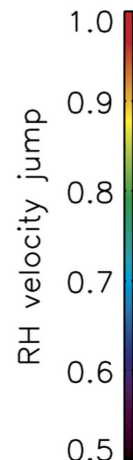

Figure 4. Type II spicules are associated with magnetoacoustic shocks passing through the corona (see arrows). From top to bottom: density, parallel velocity (along the magnetic field lines), sound speed, and maps of the estimated RH density and velocity jumps at $t=1250 \mathrm{~s}$ (right-hand side of Equations (1) and (2), respectively). The solid contour in all panels represents a temperature of $10^{5} \mathrm{~K}$. The lowest part of the spicule is situated at $x \sim 68 \mathrm{Mm}$. 
Hansteen et al. 2007; Rempel 2017). In addition, the diffusion length scales are smaller than the loop width, so they will not change the latter. Density and energy jumps become less sharp in the corona due to thermal conduction (panel (A), Figure 4), in contrast to the velocity jump (panel (B)). Therefore, the synthetic "slow" PCD intensity becomes less sharp and weaker as the perturbation propagates through the corona. In addition, flows provide mass and energy flux into the corona, which remain hot and do not return to chromospheric conditions due to strong Joule heating and thermal conduction. Hence, loops associated with spicules (panel (A) of Figure 5) are filled with mass and energy by the flows, as shown in panels (B) and (C) of Figure 5 and the associated animation and currents shown in panel (D). Both the figure and animation are made by integrating in time, the former over the lifetime of the spicule and the latter integrating forward in time from the initial formation of the spicule to the current frame, i.e., the time range shown in the top panel. This figure and the corresponding animation reveal that flows and currents are not always located exactly along the same magnetic field lines. Instead, currents and flows occur within the bulk of the magnetic field that drives the formation of the spicule. Prior to the formation of the spicule (A), we can see the remnants of a decaying hot loop. This hot loop was formed in association with a different spicule (B) that occurred earlier and at the conjugate footpoints of the field lines of spicule A. By the time of the formation of spicule A, the previous spicule had already vanished. The impact of spicule B and its associated heating is negligible in our calculations.

Let us quantify the mass and energy deposition into the corona. For this, first, we focus on the various fluxes due to advection. Averaged over the lifetime of the spicule (10 minutes), we find a net positive mass flux $\left(\sim 10^{-9} \mathrm{~g} \mathrm{~cm}^{-2} \mathrm{~s}^{-1}\right)$ and energy flux due to advection, $\left(\sim 10^{5} \mathrm{erg} \mathrm{cm}^{-2} \mathrm{~s}^{-1}\right)$ for this loop associated with the spicule (red loop located at $(x, z) \approx(57,15) \mathrm{Mm}$ in panels $(\mathrm{B})$ and $(\mathrm{C})$ of Figure 5 and the associated animation). These fluxes are measured as they cross the TR $\left(T=10^{5} \mathrm{~K}\right)$. At first glance, these numbers seem lower than the observational estimates of energy flux by an order of magnitude $\left(\sim 2 \times 10^{6} \mathrm{erg} \mathrm{cm}^{-2} \mathrm{~s}^{-1}\right.$, De Pontieu et al. 2011). Note that these numbers have been used by, e.g., Klimchuk (2012) and Klimchuk \& Bradshaw (2014). In other words, assuming a spicule diameter of $300 \mathrm{~km}$, the energy deposition due to advection in this simulation is $\sim 9 \times$ $10^{19} \mathrm{erg} \mathrm{s}^{-1}$. The discrepancy with estimates from observations is due to the following: (1) those calculations are based on assumptions driven by estimates from the observations such as constant velocity in time (100 $\mathrm{km} \mathrm{s}^{-1}$, over $2-4$ minutes), in contrast to the simulated spicule's velocity, which varies within $\sim \pm 150 \mathrm{~km} \mathrm{~s}^{-1}$ over the spicule lifetime. (2) We integrated over 10 minutes, i.e., the spicule's lifetime, whereas De Pontieu et al. (2011) integrate between 2 and 4 minutes, using the lifetime of the brightening ${ }^{8}$ or even a shorter period of time by Klimchuk (2012) and Klimchuk \& Bradshaw (2014), which gives even less total heating. (3) Last and crucially, our calculations above, including only advective terms, ignore a critical contribution to spicule energetics: the Joule heating including the dissipation of transversal wave energy.

The simulated spicules reveal the importance of the contribution coming from Joule heating $\left(3 \times 10^{-5}-10^{-2} \mathrm{erg} \mathrm{cm}^{-3} \mathrm{~s}^{-1}\right)$ shown in the bottom panels of Figures 5 and 6 . Assuming that the spicule is within $z=[3,8] \mathrm{Mm}$ and a radius of $300 \mathrm{~km}$, the

\footnotetext{
8 Note that at that time the full lifetime of spicules was unclear (Pereira et al. 2014).
}
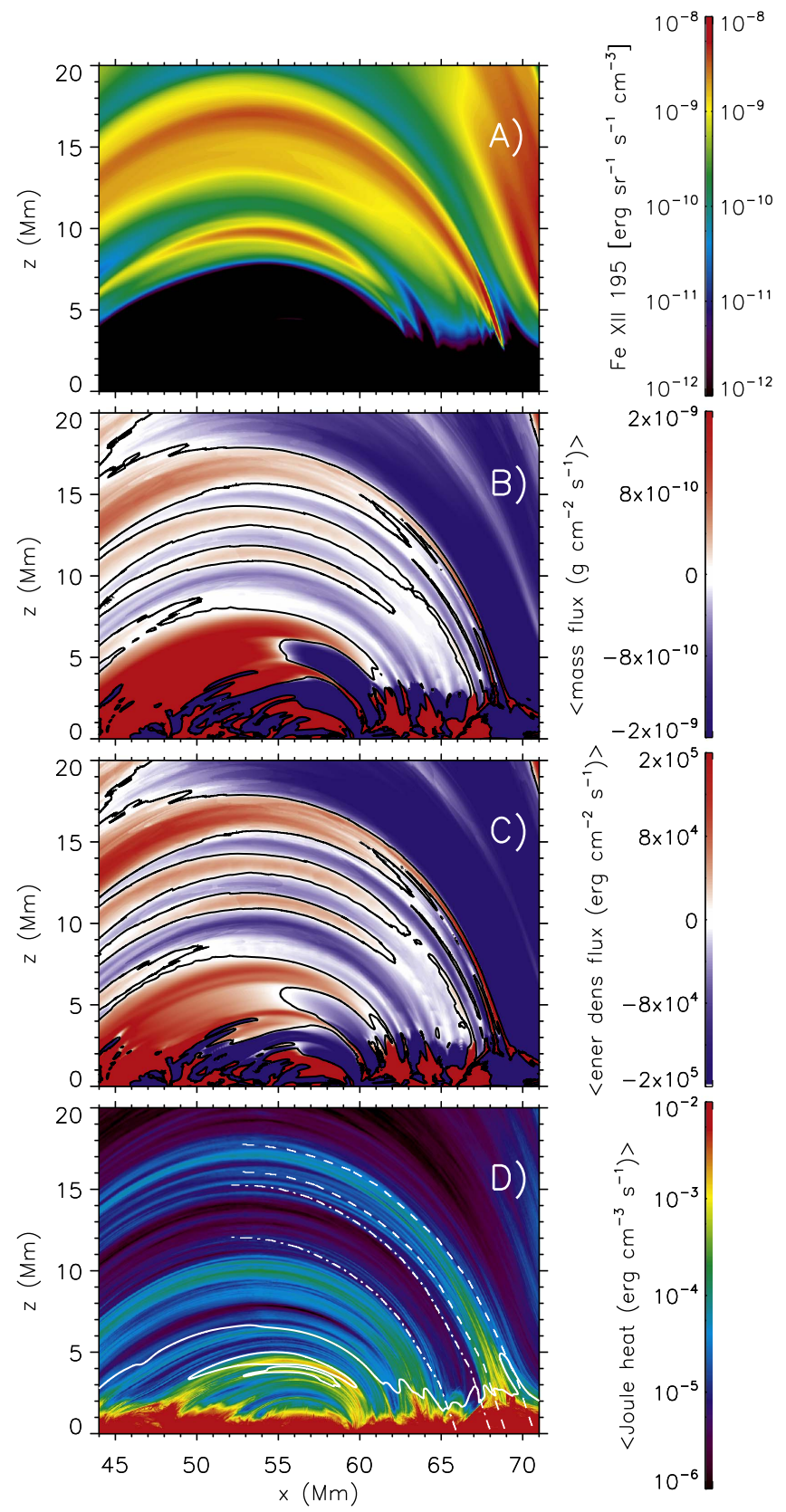

Figure 5. Type II spicules provide mass and energy density flux as well as heating to the upper atmosphere, thereby heating and filling "individual" strands. From top to bottom: Fe XII emission, mean mass, and energy flux along the magnetic field and Joule heating integrated over the lifetime of the spicule. Solid white and black contours correspond to a temperature of $10^{5} \mathrm{~K}$ and zero value, respectively. Note how the heating is mostly concentrated in magnetic field lines associated with the spicule compared to the surroundings. The dashed lines in panel (D) outline the limits used to calculate the average heating shown in Figure 6.

(An animation of this figure is available.)

energy deposition due to Joule heating is $\sim 2.4 \times 10^{22} \mathrm{erg} \mathrm{s}^{-1}$. However, the Joule heating associated with the spicule is not confined to the cold plasma of the spicule. Currents extend and propagate along the magnetic field associated with the spicule and surroundings (compare with Klimchuk 2012, who assumed that all the energy is located inside the spicule and ignored any current propagating into the corona). Taking all of this into account: the region associated with the spicule where the current plays a role (see dashed lines in Figure $6 \sim 1.5 \mathrm{Mm}$ wide and 


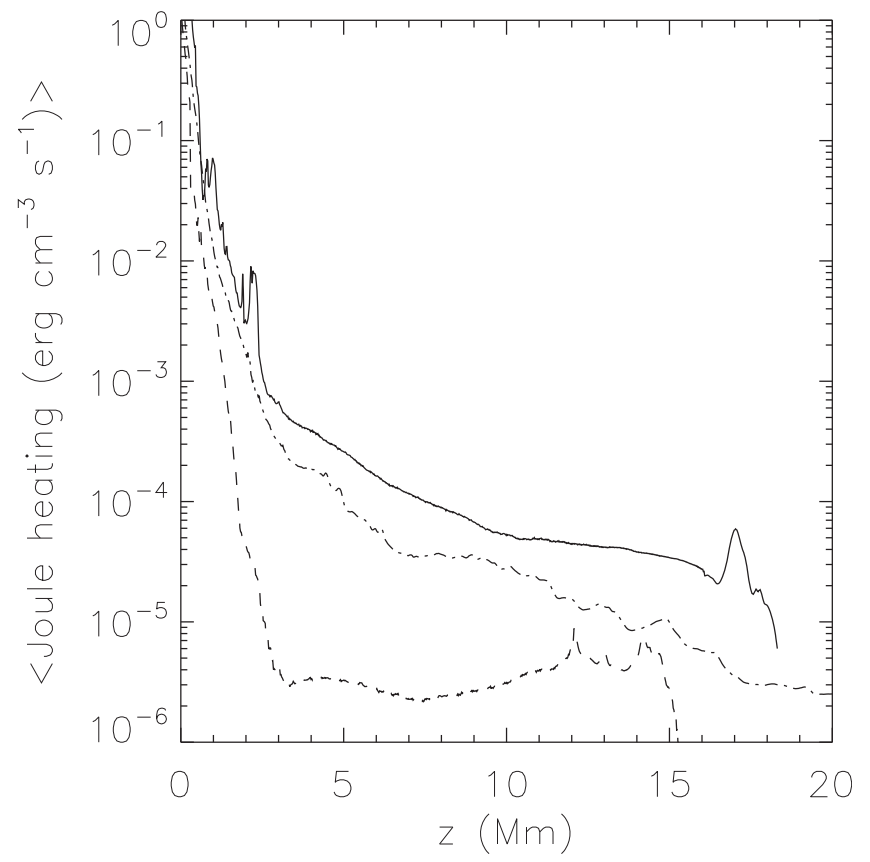

Figure 6. Mean Joule heating integrated over 10 minutes, and width as a function of height for the loop associated with the spicule $(\sim 1.5 \mathrm{Mm}$ wide, outlined by the dashed lines in panel (D) Figure 5) and without spicules ( $\sim 2.5 \mathrm{Mm}$ wide, outlined by the dotted-dashed lines in panel (D) Figure 5) are shown with solid and dashed lines respectively. For comparison, we included the mean Joule heating as a function of height for the whole simulated domain of the non-GOL simulation, i.e., without ambipolar diffusion (dotteddashed line).

$z \geqslant 3 \mathrm{Mm}$ along the associated loop), the total deposited energy due to Joule heating is $\sim 1.2 \times 10^{24} \mathrm{erg} \mathrm{s}^{-1}$, i.e., up to four orders of magnitude greater than that associated with the advective terms. Consequently, the dominant contribution to spicule heating comes from Joule heating instead of the energy flux due to kinetic and enthalpy fluxes. Taking into account the Joule heating and energy flux due to the advection of the plasma we find that this is enough to maintain the loops associated with the spicules heated to coronal temperatures for several tens of minutes.

The numbers provided above are valid for a single spicule in the model. Other spicules show similarities, but we find that the contributions from the various sources to coronal heat input can vary widely. In fact, in the model, some spicules are associated with strong heating and rather small amplitude flows and small shocks, while others have strong advection and small Joule heating. Thus, Joule heating, energy flux due to advection, and wave energy can all dominate the energy budget of individual spicules.

We find that the fast PCD in the coronal spectral lines propagates at Alfvénic speeds and is a direct consequence of Joule heating. In fact, both PCDs are associated with magnetic energy deposition in the corona, driven by the type II spicules. At the very beginning of the spicule formation, currents (and Joule heating) form along the magnetic field lines (panel (D), Figure 5 and the associated animation). These currents are driven by the release of the magnetic tension in the chromosphere, which leads to transverse waves as well as electric current propagating along the magnetic field at Alfvénic speeds. Note that transverse waves produce currents. Magnetic energy is dissipated and heats the plasma within the loop in our model by artificial (numerical) diffusion. On the Sun, one may expect a cascade to even smaller scales before dissipation sets in, e.g., via resonant absorption (Okamoto et al. 2015) of transverse waves or via the Kelvin-Helmholtz instability (Antolin et al. 2018). Although not uniform in space nor constant in time, the current is generated continuously over the lifetime of the spicule and continues to exist even after the spicule has fallen back to the chromosphere. In addition, currents do not remain in exactly the same location (or on a single magnetic field line) but "move" to those magnetic field lines that are associated with the spicule (Figure 5's animation), leading to currents "meandering" through the coronal volume within the loop associated with the spicule (see the next section for details on the width of the spicules and associated loops).

It is important to point out that the coronal Joule heating outside the loop structure associated with the spicule is, at least, an order of magnitude less than the Joule heating given by the formation of the type II spicule. This can be seen in both the bottom panel of Figure 5 and in Figure 6. In the bottom panel of Figure 5, the Joule heating inside the loop (within the white dashed lines) seen in light blue is more than an order of magnitude greater than its surroundings (e.g., see the region within the dotted-dashed lines) represented by dark blue or black. One can see a second loop structure that also has large Joule heating at roughly $x=64 \mathrm{Mm}$ associated with another type II spicule. Figure 6 shows the Joule heating as a function of height, averaged over 10 minutes and spatially limited to the loop associated with the spicule with a solid line $(\sim 1.5 \mathrm{Mm}$ wide, outlined by the dashed lines in panel (D) of Figure 5). We also show (dashed line) the Joule heating in a region that is not associated with any type II spicule ( $\sim 2.5 \mathrm{Mm}$ wide, outlined by the dotted-dashed lines in panel (D) of Figure 5). For comparison, we also show Joule heating as a function of height of the whole box for the non-GOL simulation (dotted-dashed line). Note that for regions without spicules, the Joule heating is not only lower but remains mostly constant as a function of height in the corona. We note that the average Joule heating in the non-GOL simulation is smaller than in the loops associated with the spicules (in the GOL simulation) but higher than in regions without spicules (in the GOL simulation). This is due the fact that the ambipolar diffusion reduces the amount of current that reaches into the corona in regions without spicules. It would be of great interest to expand this simulation into $3 \mathrm{D}$ in order to have a better description of the magneto-convective motions. Note that, in general, braiding is more efficient in 3D models as compared to $2 \mathrm{D}$ models and the formation of type II spicules depends on the magneto-convective motion of the magnetic field lines in the chromosphere, as pointed out in Martinez-Sykora et al. (2017), the convective motions move the magnetic field, accumulate it at the surface, and increase its tension.

Another way to visualize the heating distribution in loops associated with spicules and in loops not associated with spicules is by constructing Joule heating histograms within these two sets of loops, as shown in Figure 7. The loop associated with the spicule not only has locations with more energy but also a different slope (solid line) in comparison with the loops without type II spicules. One must be careful in assuming that the Joule heating histogram corresponds to Joule heating events (usually used in observations Benz \& Krucker 2002) since in these models it is very difficult to 


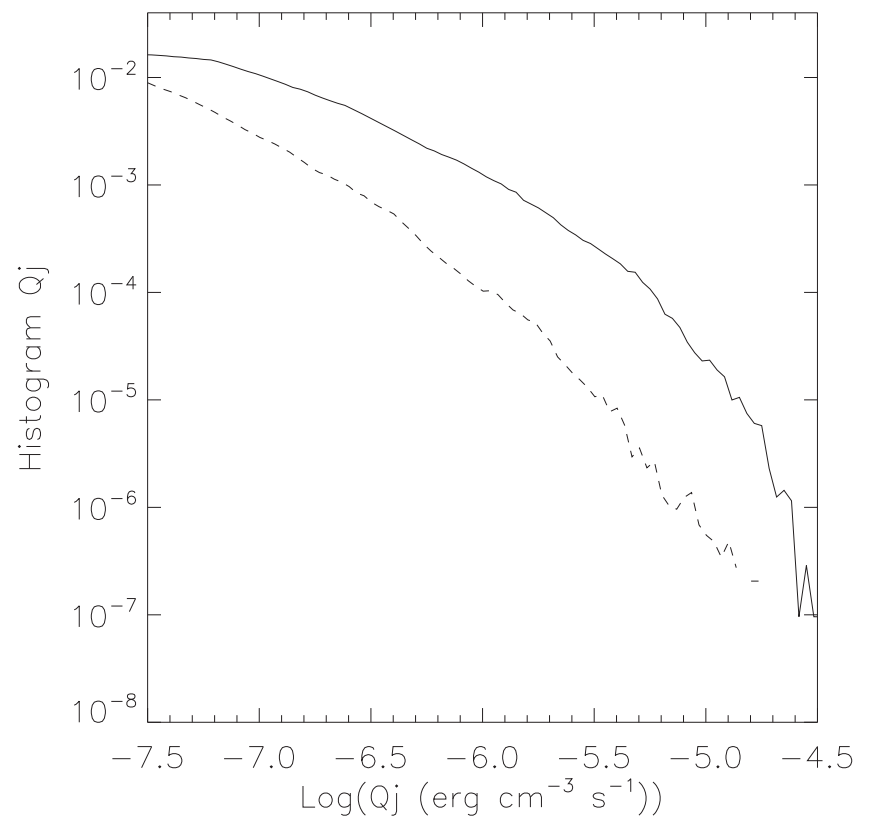

Figure 7. Joule heating histogram in the corona in the loop associated with the spicule (within the dashed lines in panel (D) in Figure 5), shown with the solid line, reaches greater values and follows a different profile than in coronal regions not affected by spicules shown with the dashed line (within the dotteddashed lines in panel (D) Figure 5).

isolate the events (Guerreiro et al. 2015; Hansteen et al. 2015; Guerreiro et al. 2017). In addition, one must interpret these results qualitatively instead of quantitatively since the simulation is only $2 \mathrm{D}$ and the magneto-convective motion and the nature of reconnection events can change when expanding into 3D.

\subsubsection{Loop Width Determined by the Processes Associated with Type II Spicule Formation}

The presence of the ambipolar diffusion and the generation of type II spicules tend to confine the propagating currents and waves to magnetic field lines associated with the spicule as shown in the previous section. The simulation without ambipolar diffusion (non-GOL) also generates transverse waves and currents due to the dynamics in the lower chromosphere. However, these currents are generated and propagate in nonspecific locations due to the magnetic convection. Consequently, the non-GOL simulation shows currents and Joule heating in extended regions in the corona, as seen in Figure 8, where many thin light-blue loops are evident in the corona (Martínez-Sykora et al. 2017 compare the global aspects of Joule heating between the non-GOL and GOL simulations). In contrast, the ambipolar diffusion confines currents to regions associated with the generation of type II spicules. The propagation of transverse waves and currents into the corona is limited to a specific set of coronal magnetic field lines as shown in light blue in panel (D) of Figure 5. In contrast, other regions have much less Joule heating (dark blue) than those field lines connected to type II spicules, as mentioned in the previous section. This leads to greater thermal contrast in the corona in the GOL simulation as compared to the non-GOL simulation, and, hence, a formation of EUV coronal loops associated with the spicules.

Similarly, the ejected mass and energy into the corona from the spicule is confined within the loop associated with the

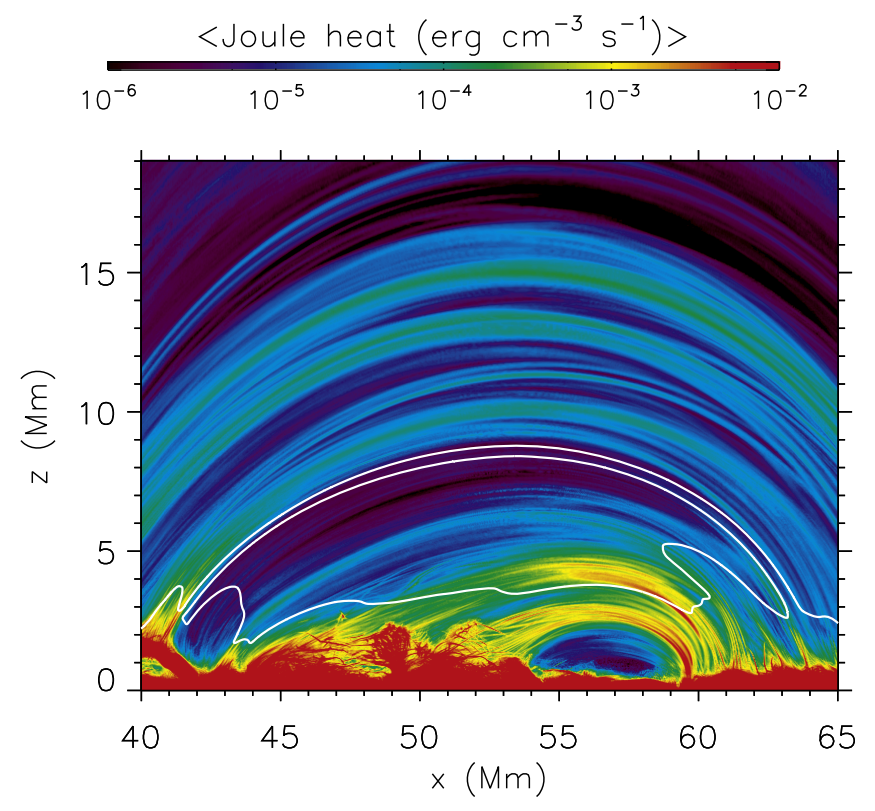

Figure 8. Non-GOL simulation shows more uniform heating distribution in contrast to the GOL simulation. Mean Joule heating integrated over the lifetime of 10 minutes is shown in logarithmic scale for the non-GOL simulation as shown for the GOL simulation in Figure 5.

spicule (see Figure 5's animation). Since the expansion of the magnetic field mostly occurs between the photosphere and middle chromosphere, in the upper chromosphere and corona the magnetic field lines barely expand. Consequently, the ejected mass and energy into the corona have roughly the same width along the loop (see Figure 5's animation). This is also clear when viewing the synthetic coronal loops shown in Figure 3. As a result of this, the loop formation due to the mass deposition is determined by the width of the spicule in the chromosphere. Consequently, the flows and currents associated with the simulated spicules are localized in loops within $500-900 \mathrm{~km}$ full width. Flows are slightly more confined $(300-600 \mathrm{~km})$ than the currents. Still, one must be aware of the 2D limitation of these simulations, which could have greater expansion.

The size of the spicule and consequently the associated coronal loop is determined by the magnetic topology at the formation of the spicule. Figure 9 shows the current perpendicular to the plane in an early state of the formation of the spicule. The driver is easy to identify and follow in time with the current (see the associated animation). In addition, we added magnetic field lines seeded (red asterisks in the animation) along the current structure that drives the spicule, i.e., the seeds do not follow the plasma nor are they forced to the same position in time. In the animation, one can see that this current is formed (built up) in the photosphere. Its length (typical in photospheric granulation, i.e., $\sim 500 \mathrm{~km}$ ) is defined due to convective motions and shapes the size of this current structure. Eventually, it penetrates into the chromosphere and drives the spicule where plasma $\beta$ is close to unity. Note that the field lines are always within the spicule, and the currents traveling into the corona last longer than the animation. During all this time, as shown in Figure 5, the current traveling into the corona is confined within the loop structure associated with the spicule, in particular, to the currents shown in Figure 9 and its animation. 


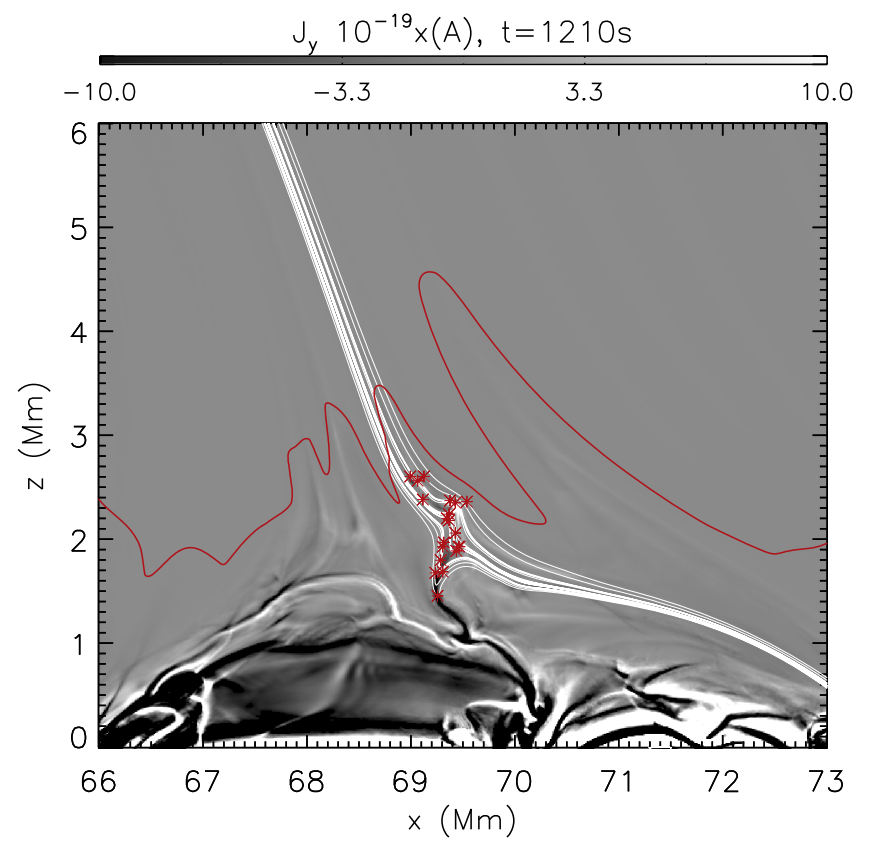

Figure 9. Current perpendicular to the plane allows us to identify the length (width) of the driver. The seeds (red asterisks) of the magnetic field lines are located along the current that drives the spicules $((x, z) \approx(69.5,1.8) \mathrm{Mm})$. The red line corresponds to temperatures of $10^{5} \mathrm{~K}$ and allows us to locate the spicule $((x, z) \approx(69,3) \mathrm{Mm})$.

(An animation of this figure is available.)

\subsection{Various Processes Throughout the Atmosphere}

In the simulated atmosphere, the Joule heating in the regions associated with the spicule follows three different power-law decays with height as shown in Figure 6. In general, the structure of the field just above the photosphere will reflect scales on the order of granulation, while at greater heights it is meso- or super-granular sized field structures that dominate. These different regimes will be reflected in the Joule dissipation of braided field lines. In particular, for the field lines associated with spicules, we have additional processes that set the scale height of magnetic field dissipation. The lower chromosphere has the steepest decay with height $(\sim-1)$, then the upper chromosphere $(\sim-1 / 5)$, and finally the least decay is in the corona $(\sim-1 / 20)$. Inside the chromosphere, this property was also observed for the ambipolar velocity as detailed in Figure 7 in Martínez-Sykora et al. (2017). There the lower and upper chromosphere are characterized by two different dominant processes that lead to different spatialand timescales. In the lower chromosphere, which is dominated by magnetoacoustic shocks, the current and Joule heating are smoother, more spatially extended than in the upper chromosphere. In contrast, in the upper chromosphere and corona, currents driven by spicules are concentrated in narrow regions along the spicules or loops. So, which process leads to different decays with height in the corona? In the simulated chromosphere $(z \approx[1,8] \mathrm{Mm})$, the Joule heating is due to ambipolar diffusion, whereas (Martínez-Sykora et al. 2017), in the corona, it is mostly due to artificial diffusion. Note that this slope strongly depends on the current that penetrates the corona coming from deeper layers (compare solid and dashed line slopes) and here, the formation of the spicules plays a crucial role. Since the model is limited to $2.5 \mathrm{D}$, type II spicules drive only transverse waves that, in a fully 3D model, may be kink or sausage waves (or other modes) and the energy deposition will vary, depending on the type of waves. Details about the transverse wave formation and energy flux are given in Martinez-Sykora et al. (2017).

\section{Discussion and Conclusions}

We report on the impact of type II spicules in the corona using 2.5D radiative MHD simulations. Our TR and coronal synthetic spectral profiles provide an explanation for the observations of so-called RB asymmetries (De Pontieu et al. 2009; Bryans et al. 2010; Peter 2010) and coronal brightenings in the $S D O /$ AIA counterpart. Our simulation is able to reproduce RB asymmetries of the same magnitude as seen in observations. This is in contrast to previous simulations that failed to reproduce observed RB asymmetries (MartínezSykora et al. 2013). All of these observational diagnostics are found to be signatures of the impact of type II spicules on the corona.

Spicules contribute to the corona through the loops that are associated with the spicules. Mass, kinetic energy flux, and Joule heating, are injected into the corona. Type II spicules are generated by the release of magnetic tension, which drives acoustic shocks and flows, and transverse waves and electric currents that travel along the magnetic field. The flows fill the loops with plasma. In addition, the temperature and density jumps are smeared out in the corona due to the thermal conduction. The RB asymmetries, line broadening, and intensity increase that occur in the early stages of the spicule are a consequence of these processes. Joule heating plays a crucial role in heating the plasma and maintaining loop temperatures against energy losses in the corona. This process leads to synthetic PCDs similar to those observed in coronal EUV imaging.

Ambipolar diffusion is a key process for the formation of type II spicules and, in addition, to localize the mass, and energy flux including waves and Joule heating in the loop associated with the spicule. All of these processes are localized within a few hundred kilometers, whereas in the surrounding corona, the amount of heating is at least an order of magnitude lower. Even if we compare with the non-GOL simulation, the heating in the simulated corona that lack spicules (in the GOL simulation) is an order of magnitude lower and the variation of Joule heating with height is almost constant, contrary to those loops associated with spicules. Since the amount of current build up in the corona via convective motion is larger in 3D than in $2 \mathrm{D}$, our results must be taken qualitatively rather than purely quantitatively. Similarly, any 3D results without ionneutral interaction effects must also be interpreted carefully.

The width of synthetic loops associated with spicules seems to be in agreement with observations (Aschwanden \& Peter 2017). Consequently, the width of coronal loops, at least for those formed in association to spicules, are not governed by micro-physics, but the macro-physics included in the model. These macro-physics determine the heating and mass deposition. The typical width of the loops associated with the spicules are set by the magnetic field topology structures formed in the photosphere. These structures drive both the currents that heat the associated loops in the corona and flows. Consequently, both flows and heating have roughly similar widths and are guided by the ambient magnetic field. 
In this model, it is very hard to separate between braiding and wave heating. In addition, note that the braiding in this model changes the classical braiding picture. Most of the magnetic tension in the photosphere and lower chromosphere is generated by convective motion. However, this magnetic energy does not propagate to greater heights easily and mostly accumulates in the lower atmosphere. Spicule formation allows the release and propagation of magnetic energy and tension to greater heights thanks to the ambipolar diffusion. Whereas, in other regions, the ambipolar diffusion dissipates magnetic energy into thermal energy within the chromosphere. As detailed here and in Martinez-Sykora et al. (2017), the released tension also drives Alfvénic waves. In the Sun, these waves may be either Alfvén waves, fast modes, kink waves, sausage modes or similar. It is important to study which modes are being generated since the Alfvénic energy flux conversion and dissipation depend on the type of the wave. Note that, since the model is limited to $2 \mathrm{D}$, the magneto-convective motion and consequently, most likely, the number of events may change when we expand this into 3D.

Last, but not least, the formation of type II spicules generates currents that propagate along the magnetic field at Alfvénic velocities into the corona. These currents are generated over the lifetime of the spicule and confined within the location of the spicule's driver but not always on the exact magnetic field lines (see the animation associated with Figure 5) containing the cold spicular material. The amount of energy contained in these currents is enough to sustain the associated coronal loops for several tens of minutes. This is in contradiction to previous findings that assumed a much smaller amount of energy release and oversimplified the heating mechanism driving the formation of type II spicules. In our model, currents are dissipated by ambipolar diffusion in the chromosphere and TR, and with artificial diffusion in the corona. Since the simulation is able to reproduce many of the observables, the artificial diffusion may mimic the unresolved physics that dissipate the sharp currents and turbulence quite well. To resolve these issues, one must perform specific studies to address further questions.

The results presented here and in Martinez-Sykora et al. (2017) are of great interest for further studies since they provide constraints for ad hoc terms that other models use, e.g., episodic heating profiles as a function of height for coronal rain simulations (Antolin et al. 2010; Xia et al. 2017), or wave amplitudes and fluxes for waves studies (Antolin et al. 2015). The heating profile shown in Figure 6 could be used as input/ driver for many other studies. However, one must take into account that the heating is localized, episodic, and not always at the exact same magnetic field lines (Figure 5's animation, Guerreiro et al. 2015; Hansteen et al. 2015).

It is important to mention that the spicules simulated here do not have a straightforward relation between the amount of mass and energy flux, wave energy, currents and Joule heating. Indeed, we found strong heating processes associated with spicules with rather low flows and small shocks, or vice versa.

We gratefully acknowledge support by NASA grants NNX16AG90G, NNH15ZDA001N, NNX17AD33G, and NNG09FA40C (IRIS) and NSF grant AST1714955. This research has received funding from the UK Science and Technology Facilities Council (Consolidated Grant ST/ K000950/1) and the European Union Horizon 2020 research and innovation programme (grant agreement No. 647214). This research was supported by the European Research Council under the European Union's Seventh Framework Programme (FP7/2007-2013)/ERC Grant agreement No. 291058. The simulations have been run on clusters from the Notur project, and the Pleiades cluster through the computing projects s1061, s1472, and s1630 from the High End Computing (HEC) division of NASA. We thankfully acknowledge the support of the Research Council of Norway through grant 230938/F50, through its Center of Excellence scheme, project number 262622, and through grants of computing time from the Programme for Supercomputing. This work has benefited from discussions at the International Space Science Institute (ISSI) meetings on "Heating of the magnetized chromosphere," where many aspects of this paper were discussed with other colleagues. To analyze the data, we have used IDL.

\section{ORCID iDs}

Juan Martínez-Sykora (ib https://orcid.org/0000-00020333-5717

Bart De Pontieu (D) https://orcid.org/0000-0002-8370-952X

Ineke De Moortel (iD https://orcid.org/0000-0002-1452-9330

Viggo H. Hansteen (1D https://orcid.org/0000-0003-0975-6659

Mats Carlsson (ii) https://orcid.org/0000-0001-9218-3139

\section{References}

Abbett, W. P., Fisher, G. H., \& Fan, Y. 2000, ApJ, 540, 548

Abramenko, V. I., Yurchyshyn, V. B., Goode, P. R., Kitiashvili, I. N., \& Kosovichev, A. G. 2012, ApJL, 756, L27

Antolin, P., Okamoto, T. J., De Pontieu, B., et al. 2015, ApJ, 809, 72

Antolin, P., Schmitt, D., Perreira, T., De Pontieu, B., \& De Moortel, I. 2018, ApJ, 856, 44

Antolin, P., Shibata, K., \& Vissers, G. 2010, ApJ, 716, 154

Aschwanden, M. J., \& Peter, H. 2017, ApJ, 840, 4

Athay, R. G., \& Holzer, T. E. 1982, ApJ, 255, 743

Beckers, J. M. 1968, SoPh, 3, 367

Benz, A. O., \& Krucker, S. 2002, ApJ, 568, 413

Braginskii, S. I. 1965, RvPP, 1, 205

Brooks, D. H., Warren, H. P., Williams, D. R., \& Watanabe, T. 2009, ApJ, 705,1522

Bryans, P., Young, P. R., \& Doschek, G. A. 2010, ApJ, 715, 1012

Carlsson, M., Hansteen, V. H., Gudiksen, B. V., Leenaarts, J., \& De Pontieu, B. 2016, A\&A, 585, A4

Carlsson, M., \& Leenaarts, J. 2012, A\&A, 539, A39

Cowling, T. G. 1957, Magnetohydrodinamics (New York: Interscience Publishers)

Culhane, J. L., Harra, L. K., James, A. M., et al. 2007, SoPh, 243, 19

De Pontieu, B., Carlsson, M., Rouppe van der Voort, L. H. M., et al. 2012, ApJL, 752, L12

De Pontieu, B., De Moortel, I., Martinez-Sykora, J., \& McIntosh, S. W. 2017, ApJL, 845, L18

De Pontieu, B., McIntosh, S., Hansteen, V. H., et al. 2007a, PASJ, 59, 655

De Pontieu, B., McIntosh, S. W., Carlsson, M., et al. 2007b, Sci, 318, 1574

De Pontieu, B., McIntosh, S. W., Carlsson, M., et al. 2011, Sci, 331, 55

De Pontieu, B., McIntosh, S. W., Hansteen, V. H., \& Schrijver, C. J. 2009, ApJL, 701, L1

De Pontieu, B., Rouppe van der Voort, L., McIntosh, S. W., et al. 2014a, Sci, 346, D315

De Pontieu, B., Title, A. M., Lemen, J. R., et al. 2014b, SoPh, 289, 2733

Galsgaard, K., \& Nordlund, A. 1996, JGR, 101, 13445

Gudiksen, B. V., \& Nordlund, A. 2005a, ApJ, 618, 1031

Gudiksen, B. V., \& Nordlund, A. 2005b, ApJ, 618, 1020

Gudiksen, B. V., Carlsson, M., Hansteen, V. H., et al. 2011, A\&A, 531, A154

Guerreiro, N., Haberreiter, M., Hansteen, V., \& Schmutz, W. 2015, ApJ, 813,61

Guerreiro, N., Haberreiter, M., Hansteen, V., \& Schmutz, W. 2017, A\&A, 603, A103

Hansteen, V., Guerreiro, N., De Pontieu, B., \& Carlsson, M. 2015, ApJ, 811,106 
Hansteen, V. H., Carlsson, M., \& Gudiksen, B. 2007, in ASP Conf. Ser. 368, 3D Numerical Models of the Chromosphere, Transition Region, and Corona, The Physics of Chromospheric Plasmas, ed. P. Heinzel, I. Dorotovič, \& R. J. Rutten (San Francisco, CA: ASP), 107

Hansteen, V. H., Hara, H., De Pontieu, B., \& Carlsson, M. 2010, ApJ, 718,1070

Hayek, W., Asplund, M., Carlsson, M., et al. 2010, A\&A, 517, A49

Klimchuk, J. A. 2012, JGRA, 117, A12102

Klimchuk, J. A., \& Bradshaw, S. J. 2014, ApJ, 791, 60

Klimchuk, J. A., Patsourakos, S., \& Tripathi, D. 2016, SoPh, 291, 55

Kobayashi, K., Cirtain, J., Winebarger, A. R., et al. 2014, SoPh, 289, 4393

Lemen, J. R., Title, A. M., Akin, D. J., et al. 2012, SoPh, 275, 17

Madjarska, M. S., Vanninathan, K., \& Doyle, J. G. 2011, A\&A, 532, L1

Mariska, J. T. 2013, SoPh, 282, 629

Martínez-Sykora, J., De Pontieu, B., Carlsson, M., et al. 2017, ApJ, 847, 36

Martínez-Sykora, J., De Pontieu, B., Hansteen, V., \& McIntosh, S. W. 2011, ApJ, 732, 84

Martinez-Sykora, J., De Pontieu, B., Hansteen, V. H., et al. 2017, Sci, 356, 1269

Martínez-Sykora, J., De Pontieu, B., Leenaarts, J., et al. 2013, ApJ, 771, 66

McIntosh, S. W., \& De Pontieu, B. 2009a, ApJ, 707, 524
McIntosh, S. W., \& De Pontieu, B. 2009b, ApJL, 706, L80

McIntosh, S. W., de Pontieu, B., Carlsson, M., et al. 2011, Natur, 475, 477

Okamoto, T. J., Antolin, P., De Pontieu, B., et al. 2015, ApJ, 809, 71

Okamoto, T. J., \& De Pontieu, B. 2011, ApJL, 736, L24

Pereira, T. M. D., De Pontieu, B., Carlsson, M., et al. 2014, ApJL, 792, L15 Peter, H. 2010, A\&A, 521, A51

Peter, H., Bingert, S., Klimchuk, J. A., et al. 2013, A\&A, 556, A104

Peter, H., Gudiksen, B. V., \& Nordlund, A. 2004, ApJL, 617, L85

Petralia, A., Reale, F., Orlando, S., \& Klimchuk, J. A. 2014, A\&A, 567, A70

Priest, E. R. 1982, Solar Magneto-Hydrodynamics (Dordrecht: Reidel)

Rempel, M. 2017, ApJ, 834, 10

Rouppe van der Voort, L., Leenaarts, J., de Pontieu, B., Carlsson, M., \& Vissers, G. 2009, ApJ, 705, 272

Schmelz, J. T., Reames, D. V., von Steiger, R., \& Basu, S. 2012, ApJ, 755, 33 Skartlien, R. 2000, ApJ, 536, 465

Skogsrud, H., Rouppe van der Voort, L., De Pontieu, B., \& Pereira, T. M. D. 2015, ApJ, 806, 170

Srivastava, A. K., Shetye, J., Murawski, K., et al. 2017, NatSR, 7, 43147

Tsiropoula, G., Tziotziou, K., Kontogiannis, I., et al. 2012, SSRv, 169, 181

Tsuneta, S., Ichimoto, K., Katsukawa, Y., et al. 2008, SoPh, 249, 167

Xia, C., Keppens, R., \& Fang, X. 2017, A\&A, 603, A42 